\title{
The Impact of Menopause on Immune Senescence
}

\author{
Flora Engelmann ${ }^{1}$ and Ilhem Messaoudi*,1,2,3 \\ ${ }^{1}$ Vaccine and Gene Therapy Institute, Oregon Health and Science University, Portland, USA \\ ${ }^{2}$ Division of Pathobiology and Immunology, Oregon National Primate Research Center, Beaverton, USA \\ ${ }^{3}$ Molecular Microbiology and Immunology, Oregon Health and Science University, Portland, USA
}

\begin{abstract}
Several lines of evidence suggest that ovarian steroids modulate immune function in women. Women are at higher risk of autoimmune disease than men; generate more robust humoral responses to vaccination than men; and plasma cytokine levels and the immune response to certain vaccines change throughout the menstrual cycle. Aging is accompanied by a decline in immunity referred to as "immune senescence" that significantly contributes to increased morbidity and mortality in the elderly. Aging is also associated with menopause, one of the most dramatic age-related physiological changes in women. Given the strong evidence for sex differences in immune function between pre-menopausal adult women and men, it has been suggested that the loss of ovarian steroids associated with menopause might contribute to decreased immune function in post-menopausal women. However, we do not yet fully understand the interplay between ovarian and immune senescence. In this article, we review studies that have investigated the impact of menopause and hormone therapy on changes in immune function.
\end{abstract}

Keywords: Immune senescence, menopause, T cells, B cells, cytokines, estrogen, progesterone.

\section{INTRODUCTION}

Data from several studies indicate that sex hormones modulate immunity. These findings suggest that the loss of ovarian steroids associated with menopause might contribute to decreased immune function observed in aged postmenopausal women. However, very few studies have examined this question. Consequently, the impact of menopause and hormone replacement therapy on immune function remains poorly understood. In this article, we begin by reviewing gender differences in the immune response to infection and vaccination. Then, we examine the effect of ovarian steroids on lymphocyte function. This is followed by an overview of the age-related decline in immunity referred to as immune senescence. Then, we summarize the effect of menopause and combined hormone therapy (HT) on immune cell frequencies and cytokine production. We conclude with a discussion of examples of diseases that illustrate the interactions between menopause and immune senescence.

\section{MODULATION OF THE IMMUNE RESPONSE TO INFECTION/VACCINATION BY OVARIAN STER- OIDS}

Data from several studies have shown sex differences in the immune response to infection (summarized in Table 1). For example, heart disease (myocarditis) after infection with coxsackievirus occurs more often in men than women [1].

\footnotetext{
*Address correspondence to this author at the Vaccine and Gene Therapy Institute Division of Pathobiology and Immunology, Oregon National Primate Research Center, Oregon Health and Science University, 505 NW $185^{\text {th }}$ Avenue, Beaverton, OR 97006, USA; Tel: 503-418-2734;

Fax: 503-418-2701; E-mail: messaoud@ohsu.edu
}

Similar sex differences are seen in a mouse model where coxsackievirus infection causes more severe disease in male and pregnant female mice as compared to non-pregnant females [2]. Moreover, viral associated disease is mitigated in castrated or estrogen treated male mice [2]. Data from murine studies suggest that estrogen promotes a Th2 type response following coxsackievirus infection rather than a CD4 Th1 cell response, which is normally seen in males and associated with the development of myocarditis [2]. Hanta virus infections are also more prevalent in men than women and this sex difference is apparent only after puberty, suggesting a protective role of ovarian steroids [2]. Adult women also generate a more robust cell mediated response than men following cytomegalovirus (CMV) infection with higher IFN $\gamma$ and interleukin (IL) -2 production [3]. Moreover, men are at greater risk for bacterial infection and sepsis after trauma, and experience greater mortality even after adjusting for age and disease severity (reviewed by Marriott et al) [4]. This difference in survival is most likely mediated by a difference in cytokine production [4]. Whereas male patients produced high levels of the Th1 cytokine tumor necrosis factor alpha (TNF $\alpha$ ) during sepsis, female patients produced higher levels of the regulatory cytokine IL-10 [5]. Similarly, males are at a greater risk for major infections after surgery, a result attributed to a higher inflammatory cytokine response [6].

Sex differences have also been detected during HIV infection. Women have been shown to have higher CD4 lymphocyte counts than men throughout infection and lower viral loads early during infection [7]. In addition, it has been found that in vitro stimulation of peripheral blood mononuclear cells (PBMC) with HIV-1 antigens that stimulate the innate immune toll-like receptor 7 (TLR-7) on plasmacytoid dendritic cells (pDCs) resulted in higher interferon- $\alpha$ (IFN $\alpha$ ) 
Table 1. Summary of Gender Differences in Outcome of Infection and Vaccination

\begin{tabular}{|c|c|c|c|}
\hline Infection & Females Compared to Males & Host & Reference \\
\hline \multicolumn{4}{|l|}{ Viral } \\
\hline \multirow[t]{2}{*}{ Coxsackievirus } & Reduced incidence of myocarditis & Human, murine & {$[1,2]$} \\
\hline & Decreased disease severity & Murine & {$[2]$} \\
\hline Hantavirus & Reduced incidence & Human, Murine & {$[2]$} \\
\hline Human Cytomegalovirus & Greater cell mediated response & & [3] \\
\hline HIV & Higher CD8 T cell and pDC activation & Human & {$[7,8]$} \\
\hline HSV-2 & Greater protection following vaccination & Human & [15-18] \\
\hline Influenza & Generate a comparable response after receiving half the vaccine dose & Human & {$[14]$} \\
\hline Hepatitis & Higher antibody response following vaccination & Human & {$[13]$} \\
\hline Bacterial & Lower risk of infection and sepsis after trauma & Human & {$[4-6]$} \\
\hline Parasitic & Reduced prevalence and disease severity & Human, murine & {$[9-11]$} \\
\hline
\end{tabular}

production by women than men [8]. This in turn, induced a stronger secondary activation of CD8+ T cells, which is consistent with HIV+ women having higher levels of CD8+ T cell activation than men with equivalent viral loads [8]. Interestingly, HIV+ postmenopausal women have lower CD4 lymphocyte counts three years after seroconversion than women of reproductive age [7], again suggesting an immuno-stimulatory effect of estradiol.

Parasitic infections also show a strong sex bias with higher prevalence of infection and disease severity in men compared to women [9]. The resistance by females to parasitic infections correlates positively with estrogen concentrations [9]. As an example of the influence of estrogen, female mice, which are less susceptible than males to Paracoccidioides brasiliensis produce Th1 type cytokines in response to infection while males produce Th2 type cytokines [10]. However, when castrated male mice are treated with $17 ß$ estradiol (E2), their spleen cells produced higher levels of IFN $\gamma$ and lower levels of IL-10 following stimulation with Plasmodium brasiliensis compared to control males, consistent with a more protective Th1 response observed in intact females [10]. Similarly, ovariectomized (OVX) mice treated with E2 produced higher levels of IFN $\gamma$ and IL-10 (associated with a protective response) as well as higher antibody responses after Plasmodium chabaudi infection compared to OVX mice treated with placebo [11]. OVX mice receiving E2 also experienced less weight and hematocrit loss, as well as less hypothermia following malaria infection than OVX mice receiving placebo [11]. Finally, E2 treatment induces resistance to Toxoplasma gondii infection in both female and male mice [9].

As described for infections, women also generate a more robust response to most vaccines as evidenced by higher rates of seroconversion and lower rates of disease after vaccination than men $[12,13]$. In an influenza vaccine trial, participants aged 18-49 and 50-64 were given either a full or half dose of vaccine. Women within the younger age group who received a half dose developed an equivalent antibody response as men who received the full dose of vaccine.
Women in the older age group receiving a half dose had slightly lower responses than men in the same age group receiving a full dose [14]. Unfortunately this study did not look at women's menopausal status, but these observations strongly suggest that the sex difference in the magnitude of the antibody response to influenza vaccine diminishes with post-menopausal status. Similarly, vaccines against HSV-2 also show sex differences in immunogenicity and efficacy. Early vaccine formulations showed some protection in women (26\%) and no protection in men (-4\%) [15]. More recently, a glycoprotein D subunit vaccine resulted in 73 to $74 \%$ protection in HSV negative women although protection in men remained negligible $(11 \%)$ [16, 17]. Data from studies using a mouse model of HSV-2 challenge strongly suggest that this gender difference in protection is mediated by E2 [18]. In contrast to intact female mice, OVX vaccinated females experienced the same rate of infection as unvaccinated controls following challenge; however, E2 treatment of intact or OVX mice enhanced protection and decreased disease severity [18]. Interestingly, antibody titers in E2 treated mice were not significantly higher than those observed in untreated mice, but the neutralization potential was significantly improved [18].

Along with the heightened immune response to vaccines, women consistently report experiencing greater adverse reactions to a number of vaccines including 17D, Influenza, MMR, and HSV2 than men [13, 19]. This would be consistent with a more vigorous immune response to the vaccine. Indeed, following Yellow Fever vaccination, gene expression of TLR-associated genes that activate the interferon pathway, was considerably higher in women than men in the first ten days after vaccination [13]. This increased gene expression would be predicted to result in higher levels of inflammation and potentially adverse reactions.

Additional data that support the modulation of immune response by female sex hormones come from cross-sectional clinical and animal studies, which show that cytokine production by peripheral blood $\mathrm{T}$ cells varies throughout the menstrual cycle. Specifically, the number of PBMC able to 
secrete IL-4 in response to phytohaemagglutinin (PHA) stimulation correlate with estrogen levels [20] and serum levels of the cytokines IL-6, IL-1 $\beta$, IL-10, and IL-8 peak during the follicular phase when estrogen levels are highest [21-23]. Moreover, several lines of evidence suggest that E2 enhances the immune response whereas progesterone dampens it. For instance, the severity of systemic lupus erythematosus (SLE) and myasthenia gravis tend to be exacerbated after E2 treatment [24, 25]. On the other hand, the severity and incidence of rheumatoid arthritis and multiple sclerosis are decreased during pregnancy [26] when circulating levels of progesterone are high.

Similar immunomodulatory observations were made during infection. Prolonged exposure to progesterone in the form of the contraceptive Depo-Provera (Depo) increases susceptibility of female mice to HSV-2 genital infection [27]. Similarly, Depo treatment increases susceptibility of nonhuman primate females to a variety of sexually transmitted diseases including Chlamydia [28], SIV [29] and SHIV [30]. Clinical studies also found associations between Depo and chlamydia, HSV-2, HIV and HPV incidence in adult women [29, 31-33]. In contrast to Depo treatment, E2 administration to ovariectomized (OVX) female mice protected them from HSV-2 infection [34]. Similarly, female rhesus macaques treated with estrogen were protected from SIV transmission [35]. Interestingly, vaccination studies in humans indicate that vaginal immunizations might be more effective for induction of genital tract antibodies if performed during the mid-follicular phase of the menstrual cycle [36]. These observations strongly suggest that progesterone inhibits whereas E2 enhances the development of protective immunity [27, 34, 37, 38].

\section{MECHANISMS OF ACTION OF OVARIAN STER- OIDS ON LYMPHOCYTE FUNCTION}

Ovarian steroids can modulate $\mathrm{T}$ and $\mathrm{B}$ cell function directly through binding of sex steroid receptors expressed by lymphocytes. Estrogen receptors are nuclear and there are two subtypes: $\alpha$ and $\beta$, which form homo and heterodimers [39]. The expression of ER $\alpha$ has been reported on lymphocytes, dendritic cells, macrophages, monocytes, natural killer cells, and mast cells ([40-46] and Fig. 1). ER expression on lymphocyte precursors is dependent on both age and stage in the cells' development [47]. For example, CD4 T cells express higher amount of $\mathrm{ER} \alpha$ than $\mathrm{ER} \beta$ while $\mathrm{B}$ cells express more ER $\beta$ than ER $\alpha$ [46]. CD8 T cells and monocytes express only low amounts of both receptor types [46]. Variation in expression levels of ERs may contribute to immune function. For instance, PBMC from SLE patients showed significantly increased levels of ER $\alpha$ mRNA and significantly decreased ER $\beta$ mRNA compared to healthy controls. Additionally, the decrease in ER $\beta$ was significantly inversely correlated to the patients' Systemic Lupus Erythematosus Disease Activity Index (SLEDAI) scores [48]. Also, as will be discussed later, the function of CD4 T cells and B cells, which have higher levels of ER expression than CD8 T cells, are also more influenced by estrogen.

Estrogen treatment of B cells increases the expression of the anti-apoptotic molecule $\mathrm{Bcl}-2$ thereby potentially increasing the resistance of auto-reactive $\mathrm{B}$ cells to apoptosis
([49-51] and Fig. 1). Estrogen also enhances B cell activation [52], IgG production [53], and upregulates activationinduced deaminase (AID), thereby enhancing somatic hypermutation frequency and class-switch recombination resulting in greater antibody affinity-maturation ([54] and Fig. 1). As described for B cells, E2 was shown to inhibit activation-induced apoptosis of T cells from SLE patients by down-regulating the expression of Fas ligand, which may allow for the persistence of autoreactive $\mathrm{T}$ cells [55]. In vitro studies examining the effect of E2 on T cell proliferation and cytokine production have often yielded contradictory results when using PBMC [56] although some observations do suggest a potential bias towards Th2, Th17 [57] and Treg polarization in E2 treated T cell cultures [58] (Fig. 1).

Ovarian steroids can also modulate adaptive immune responses indirectly by acting on cells of the innate immune system that in turn regulate the activation and differentiation of lymphocytes. For example, E2 regulates TLR2 expression on lipopolysaccharide (LPS) stimulated microglial cells in vivo [59]. While intact mice display an increased expression of TLR2 at 24 hours after both intracerebral and systemic LPS stimulation, this is not the case in OVX mice, and this outcome is reversible by the administration of E2 [59]. Further experiments using both ER $\alpha$ knock out (ERKO) and ER $\beta$ knock out (BERKO) mice, established ER $\alpha$ as the key receptor for the expression of TLR2 [59].

A recent study showed that presence of estrogen related receptor alpha $(E R R \alpha)$ was required for IFN $\gamma$ production and efficient clearance of Listeria monocytogenes by macrophages [60]. Moreover, E2 administration significantly increases mRNA for the inflammatory cytokines IL-1 $\beta$, IL-6, and TNF $\alpha$ as well as inducible NO synthase in macrophages from thioglycolate injected OVX mice via an ER $\alpha$ dependent mechanism [61]. Human dendritic cells matured in the presence of $\mathrm{E} 2$ and $\mathrm{TNF} \alpha$, but not $\mathrm{TNF} \alpha$ alone, promote the differentiation of naïve CD4 T cells into Th2 cells [62]. However, E2 matured human dendritic cells have been shown to posses a decreased capacity for stimulating $\mathrm{T}$ cell proliferation [64]. Mouse pDCs stimulated with CpG in the presence of E2 also have higher expression of the costimulatory molecules CD40 and CD86 as well as higher IFN- $\alpha$ production [63]. When co-cultured with $B$ cells, these E2 treated pDCs increase B cell viability, but not proliferative capacity [63].

Progesterone receptors (PR) are not as ubiquitous as ERs and no nuclear PR have been detected in PBMC ([39] and Fig. 1). T cells do however express membrane bound progesterone receptors $\alpha$ and $\beta$ [65]. The expression of PR $\alpha$ is also upregulated on CD8+ T cells during the luteal phase of the menstrual cycle [65]. Adding to the complexity of the effects of sex steroids, PR are primarily induced by estrogen via ER creating a complex interaction between these two hormones [39]. Natural killer cells and monocytes are the only other immune cell types to express PRs [39]. Like ER, progesterone receptors function as transcription factors by binding to progesterone responsive elements (PRE) upon ligation or by binding to other transcription factors [39]. Recent studies showed that progesterone treatment reduces the ability of dendritic cells to take up antigenic peptides, stimulate $\mathrm{T}$ cell responses [66], and secrete the potent antiviral cytokine 


\section{$\mathrm{E}$ \\ Cell Type}

$\uparrow$ Th2 51

$\uparrow$ Th17 57

$\uparrow$ Treg 58

$\uparrow \mathrm{Bcl}-2$ 49-51

$\uparrow$ Activation 52

$\uparrow \operatorname{lgg} 53$

A AID 54

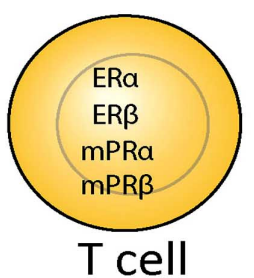

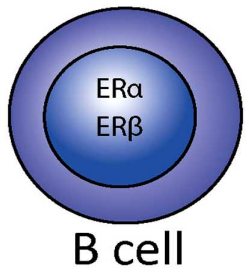

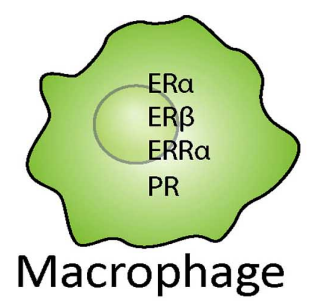

Promote Th2 62

$\downarrow$ Capacity to stimulate T cell proliferation 64

$\uparrow \mathrm{CD} 40, \mathrm{CD} 8663$

$\uparrow$ IFNY 63

$\uparrow B$ cell viability 63

\section{Unknown}
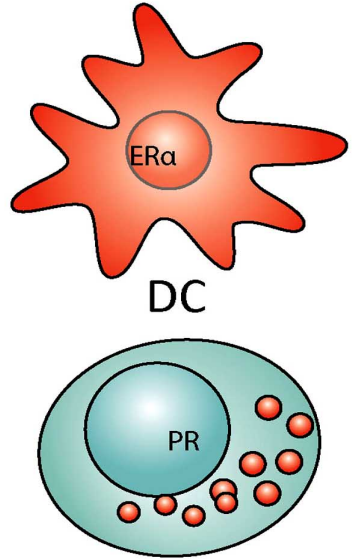

NK cells
Unknown

Unknown

Unknown

$\downarrow$ Antigenic peptide uptake 66

$\downarrow$ T cell stimulation 66

$\downarrow$ IFN $\alpha 66$

Unknown

Fig. (1). Estrogen and Progesterone receptor expression pattern and impact on immune cells.

IFN $\alpha$ [67]. Thus, as described for E2, progesterone modulates lymphocyte function both directly and indirectly by acting on innate immune cells (Fig. 1).

\section{IMMUNE SENESCENCE}

Aging is associated with a general decline in immune system function, commonly referred to as 'immune senescence,' that has been proposed as prognostic factor for human longevity [68]. This progressive deterioration affects both innate and adaptive immunity, although accumulating evidence indicate that the adaptive arm of the immune system exhibits more profound changes [69]. Longitudinal clinical studies have identified a cluster of immunological changes that are associated with immune senescence [70]. The most prominent change is a severe loss of naïve $\mathrm{T}$ cells and a shift towards memory phenotype $\mathrm{T}$ cells especially oligoclonal effector memory cells, which results in the shrinkage of the T cell repertoire and the loss of T cell proliferative ability. This loss of naïve $\mathrm{T}$ cells is driven by thymic involution and the exhaustion of naïve $T$ cells. Furthermore, aging is accompanied by a decrease in CD4/CD8 T cell ratio and a decrease in B cell numbers in PBMC [68]. Aging is also accompanied by an age-dependent upregulation of circulating pro-inflammatory cytokines, notably IL-6 and TNF $\alpha$ [71, 72]. This chronic inflammatory state is believed to significantly contribute to the development of age-related diseases such as Alzheimer's, atherosclerosis, sarcopenia, diabetes, rheumatoid arthritis and certain types of cancer [73]. 
In women, aging is accompanied by a dramatic loss in ovarian function and subsequent menopause around the age of 50 . Thus, with the average life span of $\sim 80$ years, women can expect to spend a significant portion of their lives in a post-menopausal state. The endocrine changes associated with entry into menopause appear to result from the agerelated depletion of follicular reserve. Decreasing numbers of developing follicles leads to a failure to produce the hormonal support necessary to maintain levels of inhibin $\mathrm{B}$. The decrease in inhibin B production results in deregulated production of follicular stimulating hormone (FSH), which in turn results in altered estrogen production and eventually a decrease in the levels of circulating estrogen [74]. This diminished responsiveness results in a cycle that culminates in menopause [75]. Menopause not only affects women's fertility, but also exacerbates several age-related diseases such as osteoporosis, cardiovascular disease, loss of cognitive abilities and the incidence of some cancers [76, 77].

Given that ovarian steroids modulate immune function in adult women, it is likely that the loss of ovarian steroids that occurs with menopause could have a detrimental effect on immune function in post-menopausal women $[78,79]$. This hypothesis is supported by the observation that rhinovirus infection induces a higher IFN $\gamma$ and IL-13 response in women than men, however this sex difference is no longer detected after the age of 50 [80]. Similarly, hepatitis vaccines induce higher antibody titers and seroconversion rates in adult women, but this sexual dimorphism is no longer evident in vaccinees over the age of 60 [13]. The incidence of herpes zoster is also higher in women aged 50 years of age compared to males [81, 82]. Finally, studies from our laboratory have shown that OVX female rhesus macaques generate reduced $\mathrm{T}$ and $\mathrm{B}$ cell responses to vaccination compared to age-matched intact animals. Specifically, T and B cell proliferative bursts were delayed and reduced in magnitude in OVX animals. Consequently IgG titers and frequency of IFN $\gamma+\mathrm{T}$ cells was significantly reduced in OVX animals [83].

Moreover, some of the age-related chronic diseases, notably osteoporosis and atherosclerosis, are also exacerbated by menopause [84], thereby establishing a complex interaction between immune senescence and menopause. However few studies have investigated the impact of menopause and hormone therapy on response to vaccines.

\section{THE IMPACT OF MENOPAUSE ON IMMUNE CELL FREQUENCIES}

Changes in lymphocyte numbers that occur with the onset of menopause are not entirely clear (Fig. 2). Some studies have reported a significant decline in total lymphocyte numbers [85], while others report no change [86]. However, postmenopausal HT users were found to have significantly higher lymphocyte percentages than nonusers, and new users

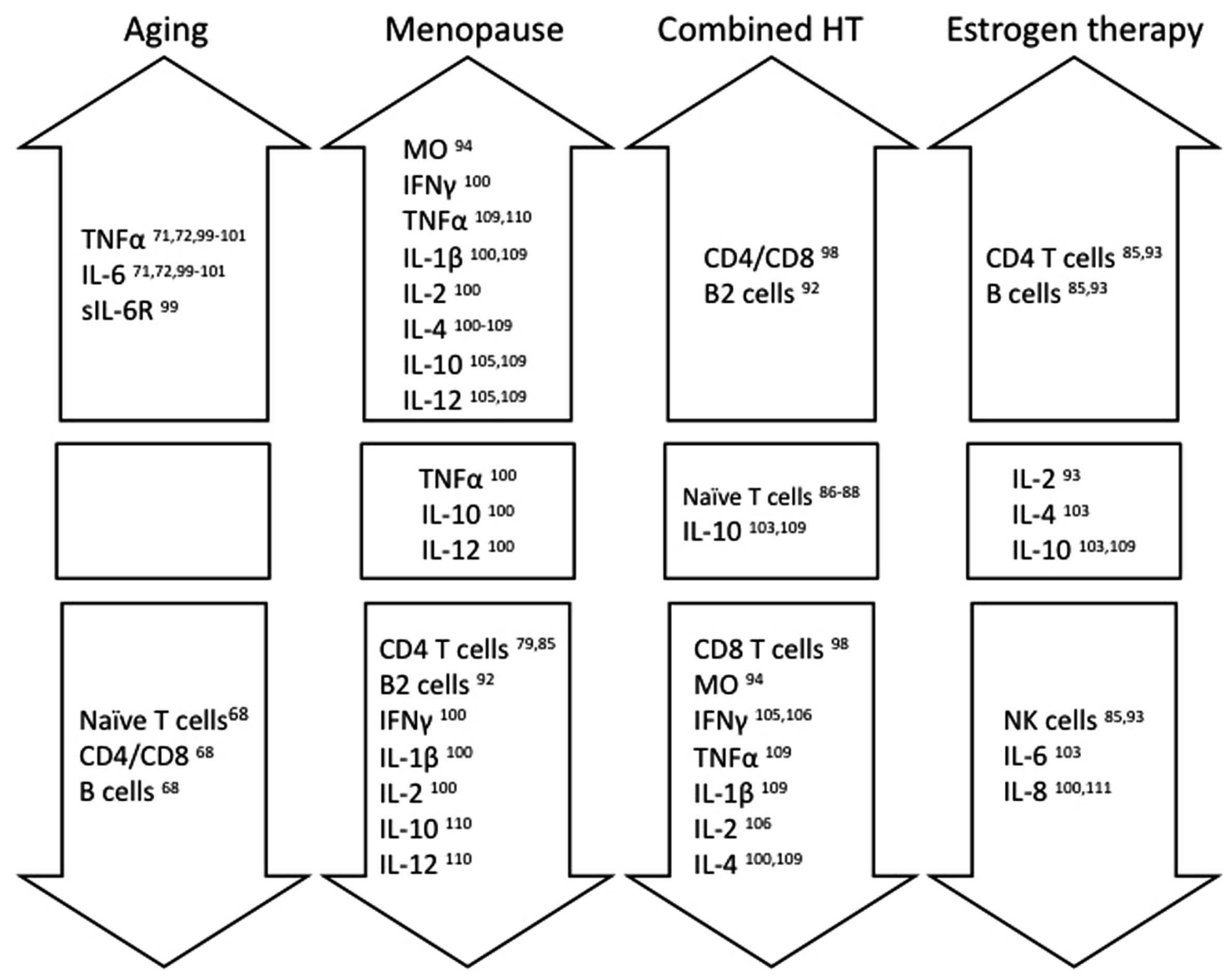

Fig. (2). Modulation of immune cell frequency and cytokine production/levels by age, menopause and hormone replacement therapy. 
experienced a significant increase in their lymphocyte percentage after just one to six months on HT [86].

One of the most conserved age-related changes is the decrease in the percentage of naïve T cells and the accumulation of memory $\mathrm{T}$ cells. There is significant decrease in naïve $\mathrm{T}$ cells and an increase in memory and activated $\mathrm{T}$ cells between early and late menopause and the use of HT has no effect on these changes, suggesting that chronological age has a more significant impact on loss of naïve $T$ cells than menopause [86-88]. Another hallmark of T cell senescence is a reduced CD4/CD8 ratio [68]. Data from a few studies suggest that menopause decreases the CD4/CD8 ratio by decreasing the frequency of CD4 T cells [79, 85]. Since, women of reproductive age have more CD4 T cells and respond more vigorously to infection/vaccination than men [89, 90], menopause-associated loss of CD4 T cells could be one of the mechanisms by which ovarian senescence contributes to immune senescence.

Total B cell numbers also decline with age and with menopause [85]. B cells can be broadly divided into B1 and $\mathrm{B} 2$ cells and their frequency is altered with increasing age [91]. B1 cells produce predominantly non-specific binding IgM whereas B2 cells, or conventional B cells are involved in the adaptive humoral immune response. The reduction in $\mathrm{B}$ cell numbers through menopause appears to be isolated to the B2 cells, which are significantly lower in late menopause compared to early and perimenopause [92]. Furthermore, B2 cells are significantly higher in HT users than non-users [92]. These studies suggest that menopause leads to a reduced humoral response and this change can potentially explain the disappearance of sex differences in antibody responses following infection and vaccination $[13,14,80]$. Similarly, total abdominal hysterectomy and oopherectomy in adult women results in a decrease in circulating B cells and CD4/CD8 ratio, and an increase in the percentage of NK cells [93]. All these changes are consistent with immune senescent phenotype.

The number of NK cells do not appear to be affected by natural menopause, but do increase with premature menopause [85] and surgical menopause [93] potentially as a compensatory mechanisms for $\mathrm{T}$ cell associated changes. Peripheral blood monocytes increase after menopause [94], but the number of tissue specific macrophages in the ovary diminish [95, 96]. To our knowledge, frequency and function of dendritic cells before and after menopause have not been studied.

Several laboratories investigated whether hormone therapy could reverse changes in circulating lymphocyte frequencies observed in post-menopausal women. Estrogen therapy reverses the decrease in CD4 and B cells and the increase in NK cells that is seen in patients who have undergone a hysterectomy [85, 93]. Similarly, combined hormone therapy reverses the age-related decrease in number of circulating $\mathrm{B}$ cells and $\mathrm{T}$ cell proliferative potential in post-menopausal women [97] and leads to an increase in B2 B cells [92]. Furthermore, estrogen has been shown to decrease the number of CD8 T cells in post-menopausal women thereby increasing the CD4/CD8 ratio [98], and to restore the levels of circulating monocytes to levels seen in cycling women [94].

\section{MODULATION OF CYTOKINE LEVELS AND PRO- DUCTION BY MENOPAUSE AND HORMONE THERAPY}

The impacts of menopause and hormone therapy on cytokine production and plasma levels are equally complex (Fig. 2). Aging is associated with an increase in circulating inflammatory cytokine levels notably IL-6 and TNF $\alpha$, a process often referred to as inflamm-aging, and believed to contribute to the development of several chronic diseases such as sarcopenia, Alzheimer's, osteoporosis and certain types of cancer [99-101]. In one cross-sectional study both IL-6 and soluble IL-6 receptor were significantly higher in postmenopausal than premenopausal women and median IL-6 levels were found to be tenfold higher in centenarians than premenopausal women [99]. Similarly, IL-6 production after in vitro stimulation also increases with age. More specifically, in vitro stimulation of PBMC with LPS shows the highest production of IL-6 as well as TNF $\alpha$ and IL-1 $\beta$ in women aged 52 to 63 as compared to young adult women [101]. Interestingly, IL-6 production by LPS stimulated PBMC is higher in women taking an estrogen plus continuous progestin regimen but not in women receiving estrogen only, compared to nonusers [102]. Similarly, women receiving transdermal estrogen experienced a significant decrease in IL-6 serum levels after three months of treatment compared to post-menopausal women who did not [103]. Indeed, serum IL-6 levels show a negative correlation with serum estrogen levels in users [103] and in women spanning the transitional stages of menopause aged 40 to 65 years [100].

A trend towards an increase in serum IFN $\gamma$ levels during early menopause ( $<5$ years post menopause) followed by a slight decrease in late menopause has been reported [100]. Similarly, IFN $\gamma$ production in whole blood or PBMC in response to either PHA or LPS stimulation in vitro, begins to increase at around the age of 40 and peaks during early to mid menopause before again decreasing during late menopause [104-106]. Previous in vitro studies have shown that estrogen has a biphasic effect on IFN $\gamma$ production by LPS stimulated whole blood samples, with low levels of estrogen stimulating and high levels inhibiting production [107]. Therefore, it is possible that as estrogen levels decrease during early menopause, it stimulates an increase IFN $\gamma$ production before becoming too low during menopause to have an effect [108]. IFN $\gamma$ serum levels decreased in perimenopausal women who have had a bilateral salpingo-oopherectomy and increase once estrogen treatment is initiated [93]. On the other hand, combined hormone therapy is associated with lower IFN $\gamma$ production probably due to the opposing effect of progesterone $[105,106]$.

A transient increase in serum IL-2 occurs in women within the first five years of menopause has been described and the data suggest a weak negative correlation with serum estrogen levels [100]. Similarly, IL-2 production following LPS stimulation of whole blood cultures increases with age, peaking during early menopause, and then declines [105]. HT reduces plasma IL-2 levels, albeit not significantly as well as IL-2 production by T cells following stimulation of purified PBMC with PHA, an approach that specifically targets T cells [106]. Another study found that transdermal administration of estrogen does not change IL-2 plasma levels 
[103]. The difference in the impact of menopause on plasma IL-2 levels between these studies may have been a result of the route of estradiol administration. Transdermal administration may not increase estrogen plasma levels enough to have an effect on IL-2 production. Alternatively, increased IL-2 levels following oral administration of estrogen could be due to hepatic first-pass effects such as increased CRP levels, which are not observed during transdermal administration of estradiol. IL-4 plasma levels were reported to increase after menopause and HT reverses this increase [100, 109]. Conversely, oopherectomy decreases IL-4 levels but ET did not affect this decrease [93]. In PHA stimulated whole blood IL-4 production does not increase until mid menopause and then becomes significantly lower in late menopause [105].

Serum TNF $\alpha$ levels were reported to increase after menopause in some studies $[109,110]$ while other studies reported no changes in serum TNF $\alpha$ levels [100]. HT reduced TNF $\alpha$ levels [109]. Similarly, data from some studies suggest that menopause is associated with increased plasma IL$1 \beta$ levels and that HT reverses this increase [109], while other studies report only a transient increase in circulating IL-1 $\beta$ [100]. The impact of menopause on IL-10 and IL-12 is equally controversial with some studies reporting an increase $[105,109]$, while others report no change [100] or a decrease in these cytokines [110]. HT and transdermal estrogen do not seem to have an impact on IL-10 levels [103, 109]. A negative correlation of IL-8 with estrogen level was reported in both humans and mice $[100,111]$.

\section{INTERPLAY BETWEEN OVARIAN AND IMMUNE SENESCENCE AND OSTEOPOROSIS}

It has long been known that post-menopausal osteoporosis is estrogen dependent. It is only during the last decade however, that the role of the adaptive immune system in this process has been discovered. It is part of a complex chain of events that begins with estrogen deprivation resulting from the cessation of ovarian function. Estrogen bound to its receptor can target estrogen receptor elements in the promoter region of some genes, thereby modulating their expression [112]. One such gene is transforming growth factor beta (TGF- $\beta$ ), a well-known negative regulator of immune function [113]. Estrogen deprivation results in reduced transcription of TGF- $\beta$, which in turn leads to increased T cell proliferation and differentiation [114], and increased levels of TNF $\alpha$ and IL-1, two key players in bone loss $[115,116]$.

Moreover, increased $\mathrm{T}$ cell activity results in elevated levels of IFN- $\gamma$ [114, 117], which activates MHC class II transactivator (CIITA) leading to greater expression of MHCII on antigen presenting cells [113, 118]. Greater numbers of memory cross-reactive CD4 T cells are thereby activated [118] resulting in increased TNF $\alpha$ production within the bone marrow and not in PBMC [113, 118, 119]. The increase in IFN- $\gamma$ levels under the condition of estrogen deficiency promotes osteoclasts due to the presence of higher levels of TNF $\alpha$, [117] which increase receptor activator of nuclear factor kappa-B ligand (RANKL) and macrophage colony-stimulating factor (M-CSF) expression as well as directly stimulate osteoclast activity [113, 118].

Another cytokine that increases dramatically with estrogen deprivation is IL-7 due in part to lower levels of TGF- $\beta$ [118]. IL-7's role in osteoporosis has been controversial. This may be because as with IFN- $\gamma$, the effects are indirect through action on $\mathrm{T}$ cell proliferation. Indeed, $\mathrm{T}$ cell deficient mice are protected from OVX induced bone loss [120] and Toraldo et al., showed IL-7 to induce OVX bone loss through a T cell dependent manner in vivo [121]. Ryan et al. take things a step further showing IL-7 increase of thymic T cell output also contributes to estrogen deficient bone loss [122].

In summary, estrogen deficient bone loss is the result of complex and often redundant pathways involving many dif-

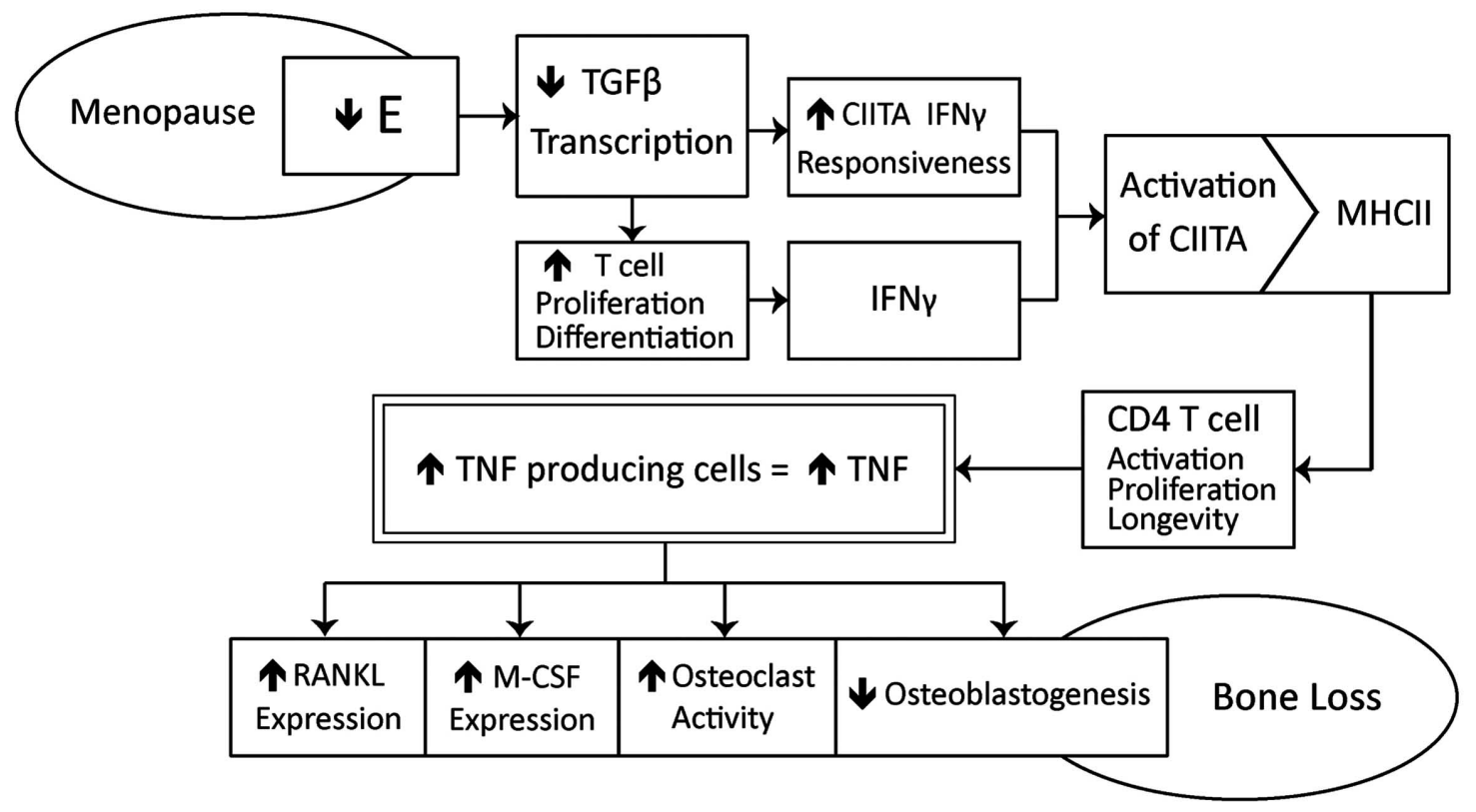

Fig. (3). The interplay of menopause and immune senescence in osteoporosis. 
ferent cytokines that offer many opportunities for potential therapies (Fig. 3). However, to date HT is the only well established effective treatment. Interestingly, studies by Vural and colleagues found that just two months of HT decreased plasma TNF $\alpha$ and IL-1b levels, and urinary hydroxyproline and calcium excretion to a pre-menopausal level [109]. These observations suggest that the mechanism by which HT exerts its bone protective effect is by reducing inflammatory cytokine production. Since menopause contributes to inflammaging, it may over time increase the expression of osteoclastogenic cytokines by T cells, which in turn exacerbates postmenopausal osteoporosis. This hypothesis that inflammaging is "responsible in great part for postmenopausal osteoporosis" was recently put forth and highlights the complex interplay between immune and ovarian senescence [123].

\section{CANCER INCIDENCE CAN BE MODULATED BY MENOPAUSE}

Risk for some cancers in women also change with the onset of menopause [124, 125]. One such cancer is hepatocellular carcinoma (HCC), a complication of chronic hepatitis C (HepC) infection [126]. Men are 3 to 5 times as likely to develop HCC than women, [126] and progression of HepC associated fibroses, a risk factor for HCC, is also higher in men as well as post-menopausal women not receiving HT compared to pre-menopausal women and postmenopausal women receiving HT [127, 128]. Naugler et al. showed that estrogen had a protective effect in male mice against chemically induced HCC and that the lack of ER $\alpha$ increased injury in female mice [126]. Humans that develop HepC cirrhotic associated HCC are more likely to have low hepatic levels of ER [129]. Estrogen inhibits IL-6 and this appears to be the key mechanism of protection [126]. In humans, both circulating and intrahepatic levels of IL-6 are higher in men and postmenopausal women compared to premenopausal women [130]. Additionally, HCC incidence was decreased and survival time increased in male IL-6 KO mice [126]. Additional studies suggest that HT may also reduce the risk of colorectal cancer [131].

\section{CONCLUDING REMARKS}

Although improvements in healthcare have led to an increase in lifespan, the age at which women enter menopause has remained relatively constant at approximately 50 years of age. Thus, with an average life span of about 80 years, women can expect to spend over one third of their lives in a post-menopausal state. Ovarian steroids have a regulatory function in several body systems and the menopause-induced decline in the levels of these steroids is likely to trigger many pathophysiological changes. One such system is the immune system, which itself undergoes significant remodeling with age. Data from several studies suggest that both natural and surgical menopause are associated with the development of immunosenescent changes and that hormone therapy can delay and/or reverse some of these changes (decreased CD4:CD8 ratio, decreased B cell frequencies and increased inflammatory cytokine levels), which in theory should result in improved immunity in post-menopausal elderly women. However, our understanding of the interplay between ovarian and immune senescence remains rather limited. A better understanding of the pleiotropic effects of ovarian steroids on immune function has important implications for women's health. Therefore more careful studies that take into account the stage of menopause in addition to other physiological parameters such as metabolic syndrome should be conducted to address this critical question.

\section{CONFLICT OF INTEREST}

None declared.

\section{ACKNOWLEDGEMENTS}

None declared.

\section{REFERENCES}

[1] Woodruff JF. Viral myocarditis. A review. Am J Pathol 1980; 101(2): 425-84.

[2] Klein SHS. Sex differences in susceptibility to viral infection, In: Klein SRC, Ed. Sex hormones and immmunity to infection. Berlin/Heidelberg: Springer 2010.

[3] Villacres MC. Predominant type 1 CMV-specific memory T-helper response in humans: evidence for gender differences in cytokine secretion. Hum Immunol 2004; 65(5): 476-85.

[4] Marriott I, Huet-Hudson YM. Sexual dimorphism in innate immune responses to infectious organisms. Immunol Res 2006; 34(3): 177-92.

[5] Schroder J. Gender differences in human sepsis. Arch Surg 1998; 133(11): 1200-5.

[6] Offner PJ, Moore EE, Biffl WL. Male gender is a risk factor for major infections after surgery. Arch Surg 1999; 134(9): 935-8.

[7] van Benthem BH. The impact of pregnancy and menopause on CD4 lymphocyte counts in HIV-infected women. AIDS 2002; 16(6): 919-24.

[8] Meier A. Sex differences in the Toll-like receptor-mediated response of plasmacytoid dendritic cells to HIV-1. Nat Med 2009; 15(8): 955-9.

[9] Klein SL. Hormonal and immunological mechanisms mediating sex differences in parasite infection. Parasite Immunol 2004; 26(67): 247-64.

[10] Pinzan CF. Immunological basis for the gender differences in murine Paracoccidioides brasiliensis infection. PLoS ONE 2010; 5(5): e10757.

[11] Klein PW. Estrogen and progesterone affect responses to malaria infection in female C57BL/6 mice. Gend Med 2008; 5(4): 423-33.

[12] Cook IF. Sexual dimorphism of humoral immunity with human vaccines. Vaccine 2008; 26(29-30): 3551-5.

[13] Klein SL, Jedlicka A, Pekosz A. The Xs and Y of immune responses to viral vaccines. Lancet Infect Dis 2010; 10(5): 338-49.

[14] Engler RJ. Half- vs full-dose trivalent inactivated influenza vaccine (2004-2005): age, dose, and sex effects on immune responses. Arch Intern Med 2008; 168(22): 2405-14.

[15] Corey L. Recombinant glycoprotein vaccine for the prevention of genital HSV-2 infection: two randomized controlled trials. Chiron HSV Vaccine Study Group. JAMA 1999; 282(4): 331-40.

[16] Bernstein DI. Safety and immunogenicity of glycoprotein Dadjuvant genital herpes vaccine. Clin Infect Dis 2005; 40(9): 127181.

[17] Stanberry LR. Glycoprotein-D-adjuvant vaccine to prevent genital herpes. N Engl J Med 2002; 347(21): 1652-61.

[18] Pennock JW, Stegall R, Bell B, et al. Estradiol improves genital herpes vaccine efficacy in mice. Vaccine 2009; 27(42): 5830-6.

[19] Pittman PR. Aluminum-containing vaccine associated adverse events: role of route of administration and gender. Vaccine 2002; 20 (Suppl 3): S48-50.

[20] Verthelyi D, Klinman DM. Sex hormone levels correlate with the activity of cytokine-secreting cells in vivo. Immunology 2000; 100(3): 384-90.

[21] Angstwurm MW, Gartner R, Ziegler-Heitbrock HW. Cyclic plasma IL-6 levels during normal menstrual cycle. Cytokine 1997; 9(5): $370-4$. 
[22] Al-Harthi L. The impact of the ovulatory cycle on cytokine production: evaluation of systemic, cervicovaginal, and salivary compartments. J Interferon Cytokine Res 2000; 20(8): 719-24.

[23] Franklin RD, Kutteh WH. Characterization of immunoglobulins and cytokines in human cervical mucus: influence of exogenous and endogenous hormones. J Reprod Immunol 1999; 42(2): 93106.

[24] Carlsten H. Estrogen accelerates immune complex glomerulonephritis but ameliorates $\mathrm{T}$ cell-mediated vasculitis and sialadenitis in autoimmune MRL lpr/lpr mice. Cell Immunol 1992; 144(1): 190-202.

[25] Delpy L. Estrogen enhances susceptibility to experimental autoimmune myasthenia gravis by promoting type 1-polarized immune responses. J Immunol 2005; 175(8): 5050-7.

[26] Confavreux C. Rate of pregnancy-related relapse in multiple sclerosis. Pregnancy in Multiple Sclerosis Group. N Engl J Med 1998; 339(5): 285-91.

[27] Gillgrass AE. Prolonged exposure to progesterone prevents induction of protective mucosal responses following intravaginal immunization with attenuated herpes simplex virus type 2. J Virol 2003; 77(18): 9845-51.

[28] Kaushic C. Chlamydia trachomatis infection in the female reproductive tract of the rat: influence of progesterone on infectivity and immune response. Infect Immun 1998; 66(3): 893-8.

[29] Marx PA. Progesterone implants enhance SIV vaginal transmission and early virus load. Nat Med 1996; 2(10): 1084-9.

[30] Trunova N. Progestin-based contraceptive suppresses cellular immune responses in SHIV-infected rhesus macaques. Virology 2006; 352(1): 169-77.

[31] Baeten JM. Hormonal contraception and risk of sexually transmitted disease acquisition: results from a prospective study. Am J Obstet Gynecol 2001; 185(2): 380-5.

[32] Brabin L. Interactions of the female hormonal environment, susceptibility to viral infections, and disease progression. AIDS Patient Care STDS 2002; 16(5): 211-21.

[33] Morrison CS. Hormonal contraception and the risk of HIV acquisition. AIDS 2007; 21(1): 85-95.

[34] Gillgrass AE. Estradiol regulates susceptibility following primary exposure to genital herpes simplex virus type 2, while progesterone induces inflammation. J Virol 2005; 79(5): 3107-16.

[35] Smith SM, Baskin GB, Marx PA. Estrogen protects against vaginal transmission of simian immunodeficiency virus. J Infect Dis 2000; 182(3): 708-15.

[36] Kozlowski PA. Differential induction of mucosal and systemic antibody responses in women after nasal, rectal, or vaginal immunization: influence of the menstrual cycle. J Immunol 2002; 169(1): 566-74.

[37] Kaushic C. Progesterone increases susceptibility and decreases immune responses to genital herpes infection. J Virol 2003; 77(8): 4558-65.

[38] Abel K. Abrogation of attenuated lentivirus-induced protection in rhesus macaques by administration of depo-provera before intravaginal challenge with simian immunodeficiency virus mac239. J Infect Dis 2004; 190(9): 1697-705.

[39] Kovats SEC, Hemant A. Sex steroid receptors in immune cells. In: Klevin SL, Ed. Sex hormones and immunity to infection. Berlin/Heidelberg: Springer 2010.

[40] Curran EM. Natural killer cells express estrogen receptor-alpha and estrogen receptor-beta and can respond to estrogen via a nonestrogen receptor-alpha-mediated pathway. Cell Immunol 2001; 214(1): 12-20.

[41] Grimaldi CM. Estrogen alters thresholds for B cell apoptosis and activation. J Clin Invest 2002; 109(12): 1625-33.

[42] Harkonen PL, Vaananen HK. Monocyte-macrophage system as a target for estrogen and selective estrogen receptor modulators. Ann N Y Acad Sci 2006; 1089: 218-27.

[43] Komi J, Lassila O. Nonsteroidal anti-estrogens inhibit the functional differentiation of human monocyte-derived dendritic cells. Blood 2000; 95(9): 2875-82.

[44] Mor G. Interaction of the estrogen receptors with the Fas ligand promoter in human monocytes. J Immunol 2003; 170(1): 114-22.

[45] Paharkova-Vatchkova V, Maldonado R, Kovats S. Estrogen preferentially promotes the differentiation of CD11c+ CD11b(intermediate) dendritic cells from bone marrow precursors. J Immunol 2004; 172(3): 1426-36.
[46] Phiel KL. Differential estrogen receptor gene expression in human peripheral blood mononuclear cell populations. Immunol Lett 2005; 97(1): 107-13.

[47] Igarashi H. Age and stage dependency of estrogen receptor expression by lymphocyte precursors. Proc Natl Acad Sci U S A 2001; 98(26): 15131-6.

[48] Inui A. Estrogen receptor expression by peripheral blood mononuclear cells of patients with systemic lupus erythematosus. Clin Rheumatol 2007; 26(10): 1675-8.

[49] Verthelyi DI, Ahmed SA. Estrogen increases the number of plasma cells and enhances their autoantibody production in nonautoimmune C57BL/6 mice. Cell Immunol 1998; 189(2): 12534.

[50] Rider V. Estrogen increases CD40 ligand expression in T cells from women with systemic lupus erythematosus. J Rheumatol 2001; 28(12): 2644-9.

[51] Evans MJ. Estrogen decreases in vitro apoptosis of peripheral blood mononuclear cells from women with normal menstrual cycles and decreases TNF-alpha production in SLE but not in normal cultures. Clin Immunol Immunopathol 1997; 82(3): 258-62.

[52] Paavonen T, Andersson LC, Adlercreutz H. Sex hormone regulation of in vitro immune response. Estradiol enhances human B cell maturation via inhibition of suppressor $T$ cells in pokeweed mitogen-stimulated cultures. J Exp Med 1981; 154(6): 1935-45.

[53] Kanda N, Tamaki K. Estrogen enhances immunoglobulin production by human PBMCs. J Allergy Clin Immunol 1999; 103(2 Pt 1): 282-8.

[54] Karpuzoglu E, Zouali M. The multi-faceted influences of estrogen on lymphocytes: toward novel immuno-interventions strategies for autoimmunity management. Clin Rev Allergy Immunol 2011; 40(1): $16-26$.

[55] Kim WU. Effect of oestrogen on T cell apoptosis in patients with systemic lupus erythematosus. Clin Exp Immunol 2010; 161(3): 453-8.

[56] Bouman A, Heineman MJ, Faas MM. Sex hormones and the immune response in humans. Hum Reprod Update 2005; 11(4): 411-23.

[57] Polanczyk MJ. Estrogen-mediated immunomodulation involves reduced activation of effector $\mathrm{T}$ cells, potentiation of Treg cells, and enhanced expression of the PD-1 costimulatory pathway. J Neurosci Res 2006; 84(2): 370-8.

[58] Khan D. Estrogen increases, whereas IL-27 and IFN-gamma decrease, splenocyte IL-17 production in WT mice. Eur J Immunol 2010; 40(9): 2549-56.

[59] Soucy G. Estradiol is required for a proper immune response to bacterial and viral pathogens in the female brain. J Immunol 2005; 174(10): 6391-8.

[60] Sonoda J. Nuclear receptor ERR alpha and coactivator PGC-1 beta are effectors of IFN-gamma-induced host defense. Genes Dev 2007; 21(15): 1909-20.

[61] Calippe B. 17\{beta\}-Estradiol promotes TLR4-triggered proinflammatory mediator production through direct estrogen receptor \{alpha\} signaling in macrophages in vivo. J Immunol 2010; 185(2): 1169-76.

[62] Uemura Y. 17 Beta-estradiol (E2) plus tumor necrosis factor-alpha induces a distorted maturation of human monocyte-derived dendritic cells and promotes their capacity to initiate T-helper 2 responses. Hum Immunol 2008; 69(3): 149-57.

[63] Li X. 17beta-estradiol enhances the response of plasmacytoid dendritic cell to CpG. PLoS One 2009; 4(12): e8412.

[64] Segerer SE. Impact of female sex hormones on the maturation and function of human dendritic cells. Am J Reprod Immunol 2009; 62(3): 165-73.

[65] Dosiou C. Expression of membrane progesterone receptors on human T lymphocytes and Jurkat cells and activation of G-proteins by progesterone. J Endocrinol 2008; 196(1): 67-77.

[66] Butts CL. Progesterone inhibits mature rat dendritic cells in a receptor-mediated fashion. Int Immunol 2007; 19(3): 287-96.

[67] Hughes GC. Cutting edge: progesterone regulates IFN-\{alpha\} production by plasmacytoid dendritic cells. J Immunol 2008; 180(4): 2029-33.

[68] Larbi A. Aging of the Immune System as a prognostic factor for human longevity. Physiology 2008; 23(2): 64-74.

[69] Pawelec G, Larbi A, Derhovanessian E. Senescence of the human immune system. J Comp Pathol 2010; 142(Suppl. 1): S39-44. 
[70] Derhovanessian E, Larbi A, Pawelec G. Biomarkers of human immunosenescence: impact of Cytomegalovirus infection. Curr Opin Immunol 2009; 21(4): 440-5.

[71] De Martinis M. Inflamm-ageing and lifelong antigenic load as major determinants of ageing rate and longevity. FEBS Lett 2005; 579(10): 2035-9.

[72] Wikby A. The immune risk phenotype is associated with IL-6 in the terminal decline stage: findings from the Swedish NONA immune longitudinal study of very late life functioning. Mech Ageing Dev 2006; 127(8): 695-704.

[73] Vasto S. Inflammatory networks in ageing, age-related diseases and longevity. Mech Ageing Dev 2007; 128(1): 83-91.

[74] Burger HG. Cycle and hormone changes during perimenopause: the key role of ovarian function. Menopause 2008; 15(4 Pt 1): 60312.

[75] Wu JM. Ovarian aging and menopause: current theories, hypotheses, and research models. Exp Biol Med (Maywood) 2005; 230(11): 818-28.

[76] Grady D. Clinical practice. Management of menopausal symptoms. N Engl J Med 2006; 355(22): 2338-47.

[77] Prior JC. Perimenopause: the complex endocrinology of the menopausal transition. Endocr Rev 1998; 19(4): 397-428.

[78] Gameiro CM, Romao F, Castelo-Branco C. Menopause and aging: changes in the immune system--a review. Maturitas 2010; 67(4): 316-20.

[79] Gameiro C, Romao F. Changes in the immune system during menopause and aging. Front Biosci (Elite Ed). 2010; 2: 1299-303.

[80] Carroll ML. Adaptive immunity to rhinoviruses: sex and age matter. Respir Res 2010; 11: 184.

[81] Chapman RS, Cross KW, Fleming DM. The incidence of shingles and its implications for vaccination policy. Vaccine 2003; 21(1920): 2541-7.

[82] Fleming DM. Gender difference in the incidence of shingles. Epidemiol Infect 2004; 132(1): 1-5.

[83] Engelmann F. Accelerated immune senescence and reduced response to vaccination in ovariectomized female rhesus macaques. Age (Dordr) 2011; 33(3): 275-89.

[84] Ginaldi L, Di Benedetto MC, De Martinis M. Osteoporosis, inflammation and ageing. Immun Ageing 2005; 2: 14.

[85] Giglio T. Immune cell circulating subsets are affected by gonadal function. Life Sci 1994; 54(18): 1305-12.

[86] Kamada M. Effect of hormone replacement therapy on postmenopausal changes of lymphocytes and $\mathrm{T}$ cell subsets. $\mathrm{J}$ Endocrinol Invest 2000; 23(6): 376-82.

[87] Fahlman MM. Effects of hormone replacement therapy on selected indices of immune function in postmenopausal women. Gynecol Obstet Invest 2000; 50(3): 189-93.

[88] Yang JH. Hormone replacement therapy reverses the decrease in natural killer cytotoxicity but does not reverse the decreases in the T-cell subpopulation or interferon-gamma production in postmenopausal women. Fertil Steril 2000; 74(2): 261-7.

[89] Maini MK. Reference ranges and sources of variability of CD4 counts in HIV-seronegative women and men. Genitourin Med 1996; 72(1): 27-31.

[90] Tollerud DJ. The influence of age, race, and gender on peripheral blood mononuclear-cell subsets in healthy nonsmokers. J Clin Immunol 1989; 9(3): 214-22.

[91] Weksler ME, Szabo The effect of age on the B-cell repertoire. J Clin Immunol 2000; 20(4): 240-9.

[92] Kamada M. B cell subsets in postmenopausal women and the effect of hormone replacement therapy. Maturitas 2001; 37(3): 173-9.

[93] Kumru S, Godekmerdan A, Yilmaz B. Immune effects of surgical menopause and estrogen replacement therapy in peri-menopausal women. J Reprod Immunol 2004; 63(1): 31-8.

[94] Ben-Hur H. Menopause is associated with a significant increase in blood monocyte number and a relative decrease in the expression of estrogen receptors in human peripheral monocytes. Am J Reprod Immunol 1995; 34(6): 363-9.

[95] Best CL. Localization and characterization of white blood cell populations within the human ovary throughout the menstrual cycle and menopause. Hum Reprod 1996; 11(4): 790-7.

[96] Katabuchi H. Distribution and fine structure of macrophages in the human ovary during the menstrual cycle, pregnancy and menopause. Endocr J 1997; 44(6): 785-95.

[97] Porter VR. Immune effects of hormone replacement therapy in post-menopausal women. Exp Gerontol 2001; 36(2): 311-26.
[98] Holl M, Donat H, Weise W. [Peripheral blood lymphocyte subpopulations of postmenopausal women with hormone replacement therapy]. Zentralbl Gynakol 2001; 123(9): 543-5.

[99] Giuliani N. Serum interleukin-6, soluble interleukin-6 receptor and soluble gp130 exhibit different patterns of age- and menopauserelated changes. Exp Gerontol 2001; 36(3): 547-57.

[100] Yasui T. Changes in serum cytokine concentrations during the menopausal transition. Maturitas 2007; 56(4): 396-403.

[101] Kim OY. Effects of aging and menopause on serum interleukin-6 levels and peripheral blood mononuclear cell cytokine production in healthy nonobese women. Age (Dordr) 2012; 34(2): 415-25.

[102] Brooks-Asplund EM. Hormonal modulation of interleukin-6, tumor necrosis factor and associated receptor secretion in postmenopausal women. Cytokine 2002; 19(4): 193-200.

[103] Saucedo R. Transdermal estradiol in menopausal women depresses interleukin-6 without affecting other markers of immune response. Gynecol Obstet Invest 2002; 53(2): 114-7.

[104] Kamada M. Transient increase in the levels of T-helper 1 cytokines in postmenopausal women and the effects of hormone replacement therapy. Gynecol Obstet Invest 2001; 52(2): 82-8.

[105] Deguchi K. Postmenopausal changes in production of type 1 and type 2 cytokines and the effects of hormone replacement therapy. Menopause 2001; 8(4): 266-73.

[106] Stopinska-Gluszak U. Effect of estrogen/progesterone hormone replacement therapy on natural killer cell cytotoxicity and immunoregulatory cytokine release by peripheral blood mononuclear cells of postmenopausal women. J Reprod Immunol 2006; 69(1): 65-75.

[107] Matalka KZ. The effect of estradiol, but not progesterone, on the production of cytokines in stimulated whole blood, is concentration-dependent. Neuro Endocrinol Lett 2003; 24(3-4): 185-91.

[108] Goetzl EJ. Gender specificity of altered human immune cytokine profiles in aging. FASEB J 2010; 24(9): 3580-9.

[109] Vural P, Canbaz M, Akgul C. Effects of menopause and postmenopausal tibolone treatment on plasma TNFalpha, IL-4, IL10, IL-12 cytokine pattern and some bone turnover markers. Pharmacol Res 2006; 53(4): 367-71.

[110] Kamada M. Postmenopausal changes in serum cytokine levels and hormone replacement therapy. Am J Obstet Gynecol 2001; 184(3): 309-14.

[111] Abu-Taha M. Menopause and ovariectomy cause a low grade of systemic inflammation that may be prevented by chronic treatment with low doses of estrogen or losartan. J Immunol 2009; 183(2): 1393-402.

[112] Yang N. Estrogen-related receptor, hERR1, modulates estrogen receptor-mediated response of human lactoferrin gene promoter. J Biol Chem 1996; 271(10): 5795-804.

[113] Pacifici R. T cells: critical bone regulators in health and disease. Bone 2010; 47(3): 461-71.

[114] Gorelik L, Flavell RA. Transforming growth factor-beta in T-cell biology. Nat Rev Immunol 2002; 2(1): 46-53.

[115] Charatcharoenwitthaya N. Effect of blockade of TNF-alpha and interleukin-1 action on bone resorption in early postmenopausal women. J Bone Miner Res 2007; 22(5): 724-9.

[116] D'Amelio Estrogen deficiency increases osteoclastogenesis upregulating T cells activity: a key mechanism in osteoporosis. Bone 2008; 43(1): 92-100.

[117] Gao Y. IFN-gamma stimulates osteoclast formation and bone loss in vivo via antigen-driven T cell activation. J Clin Invest 2007; 117(1): 122-32.

[118] Weitzmann MN, Pacifici R. Estrogen regulation of immune cell bone interactions. Ann N Y Acad Sci 2006; 1068: 256-74.

[119] Roggia C. Up-regulation of TNF-producing T cells in the bone marrow: a key mechanism by which estrogen deficiency induces bone loss in vivo. Proc Natl Acad Sci USA 2001; 98(24): 13960-5.

[120] Cenci S. Estrogen deficiency induces bone loss by enhancing T-cell production of TNF-alpha. J Clin Invest 2000; 106(10): 1229-37.

[121] Toraldo G. IL-7 induces bone loss in vivo by induction of receptor activator of nuclear factor kappa B ligand and tumor necrosis factor alpha from T cells. Proc Natl Acad Sci USA 2003; 100(1): 125-30.

[122] Ryan MR. An IL-7-dependent rebound in thymic T cell output contributes to the bone loss induced by estrogen deficiency. Proc Natl Acad Sci USA 2005; 102(46): 16735-40.

[123] Lencel P, Magne D. Inflammaging: the driving force in osteoporosis? Med Hypotheses 2011; 76(3): 317-21. 
[124] Mossa B. Occurrence of malignancy in endometrial polyps during postmenopause. Eur J Gynaecol Oncol 2010; 31(2): 165-8.

[125] Brinton LA. Reproductive and hormonal factors and lung cancer risk in the NIH-AARP diet and health study cohort. Cancer Epidemiol Biomarkers Prev 2011; 20(5): 900-11.

[126] Naugler WE. Gender disparity in liver cancer due to sex differences in MyD88-dependent IL-6 production. Science 2007; 317(5834): 121-4.

[127] Di Martino V. Progression of liver fibrosis in women infected with hepatitis C: long-term benefit of estrogen exposure. Hepatology 2004; 40(6): 1426-33.

[128] Codes L. Liver fibrosis in women with chronic hepatitis C: evidence for the negative role of the menopause and steatosis and the potential benefit of hormone replacement therapy. Gut 2007; 56(3): 390-5.

[129] Shimizu I. Estrogen receptor levels and lipid peroxidation in hepatocellular carcinoma with hepatitis $C$ virus infection. Liver 2001; 21(5): 342-9.

[130] Grivennikov SI, Karin M. Inflammatory cytokines in cancer: tumour necrosis factor and interleukin 6 take the stage. Ann Rheum Dis 2011; 70 (Suppl 1): i104-8.

[131] La Vecchia C, Brinton LA, McTiernan A. Cancer risk in menopausal women. Best Pract Res Clin Obstet Gynaecol 2002; 16(3): 293-307.

Received: June 24, 2011

Revised: August 23, 2011

Accepted: October 03, 2011

(C) Engelmann and Messaoudi; Licensee Bentham Open.

This is an open access article licensed under the terms of the Creative Commons Attribution Non-Commercial License (http://creativecommons.org/licenses/by-nc/3.0/) which permits unrestricted, non-commercial use, distribution and reproduction in any medium, provided the work is properly cited. 